fewer than two per day. ${ }^{5}$ Many Albanian women feel threatened by Serbian concerns about their high birth rate and prefer the risks of no prenatal care and home delivery. Moreover, dismissed workers and their families have no social security and thus are not entitled to free health care, so they are dependent on charities such as the Mother Theresa clinic.

I visited the clinic, housed in a small shop in an unpaved street. In an upstairs corridor Dr Myrvete Pagarada was seeing gynaecology outpatients. The queue extended into the road. "Everything comes too late-when it's severe. There's no possibility for basic prevention like cervical smears. We are back to the seventeenth century." Albanians believe that the complete disruption of the health care system has done more damage than sanctions. For example, vaccination rates have fallen in some communities from around $80 \%$ to as low as $30 \%$ in those health sectors under "special measures" (University of Prishtina medical faculty, unpublished data, March 1993).

\section{Prospects for reconciliation}

The problems in health care must be seen in the context of continuing repression of the Albanian population. While Serbian men are recruited into the local police and armed, "weapons searches" of unarmed Albanians become the excuse for further beatings and detentions. Two searches took place in the three days that I was there. Evictions of dismissed workers from tied accommodation continue. The refusal of Albanian teachers to teach the new unified Serbian school curriculum has resulted in their dismissal and the closure of schools to Albanian children. Reportedly, three parents were killed and others wounded while accompanying their children to an unofficial Albanian school. ${ }^{\circ}$ And when I asked a class of forty 17 year olds, sitting on mattresses studying Ovid in a private house, what they would do when they graduated the boys uniformly replied that they would leave Kosovo or hide, to avoid being drafted into the Yugoslav army. A total of 300000 Albanians have left in the past three years.

Albanians regard the creation of parallel systems of education and health care as a way of maintaining the human, social, and ethnic rights they enjoyed before 1989 and of non-violently defending themselves against what they see as a "quiet form of ethnic cleansing." Working Albanians and many abroad provide the financial support. And regardless of the dispute over the province's political status they would like dialogue with the Serbian authorities to resolve these practical issues. However, concerns over human rights abuses and calls for dialogue are perceived by the Serbian authorities as "a mask...to get time until wanted separatist goals are achieved," ${ }^{7}$ and little dialogue has occurred.

Dr Stevan Baljosevic, director of Prishtina hospital, told me his only concern was his patients' wellbeing. Yet it is hard to see how the best patient care can be achieved in a setting where large numbers of skilled staff are dismissed because of ethnic and political affiliations, where medicine cannot be taught in the language of most of the population, and where doctors must monitor their clinical opinions, their therapeutic interventions, and their patients' behaviour for political correctness.

A brutal war is raging in Bosnia $200 \mathrm{~km}$ away. The hope of avoiding it in Kosovo lies ultimately with Serbia finding a way to address its own needs in a manner that also addresses the needs of others. The reinstatement of unfairly dismissed health workers and the reopening of the Albanian medical school would benefit both Serbians and Albanians in the province. It might be a good way to begin. Throughout this article Kosovo is spelt in the conventional way. Albanians refer to the region as Kosova.

\footnotetext{
1 Mekuli D. Kosova health service under emergency management. Kosova Watch 1992;1:25.

2 Ralic P. Minority rights in Serbia: facts, figures, orientation. Belgrade: Ministry of Information, 1992:58.

3 Helsinki Watch interview with Kosovo Secretary for Education Miodrag Djuricic, December 1991. In: Human Rights Watch. Yuroslavia: human Djuricic, December 1991. In: Human Rights Watch. Y
rights abuses in Kosovo, 1990-1992. New York: HRW, 1992:5.

4 Human Rights Watch. Yugoslavia: human rights abuses in Kosovo, 1990-1992. New York: HRW, 1992:58.

5 Mekuli D. Kosova health service under emergency management. Kosova Watch 1992;1:1-28.

6 Amnesty International. Urgent action appeal, extra 10/92. 1992 Jan 31

7 Ralic P. Minority rights in Serbia: facts, figures, orientation. Belgrade: Ministry of Information, 1992:33.
}

Mayday Hospital, Croydon CR7 7YE

A C Miller, consultant physician

\section{Southmead Hospital,} Bristol BS10 5NB

JE Harvey, consultant physician

Correspondence to: Dr Miller.

\title{
Guidelines for the management of spontaneous pneumothorax
}

\author{
A C Miller, J E Harvey on behalf of Standards of Care Committee, British Thoracic Society
}

Simple flow diagrams, with explanatory notes, have been devised to assist in the immediate and subsequent management of patients presenting to casualty departments with spontaneous pneumothorax. They cover decision making about which patients require admission; whether a drainage procedure is necessary; if so, the appropriate method, including technical details; inpatient treatment; referral of inpatients to a respiratory specialist; and follow up arrangements. The guidelines, designed for incorporation into casualty and ward handbooks, have already proved valuable in several district general hospitals, and can be used as a basis for audit.

Spontaneous pneumothorax is common in two groups of patients: otherwise healthy young adults, who can tolerate a large air leak, and older patients with emphysema, in whom even a small pneumothorax may cause severe respiratory failure. A district general hospital with a catchment population of 200000 may expect to treat 25 such patients a year, so all casualty doctors and general physicians need to be familiar with the management of this condition.

Although intercostal drainage has been standard treatment for pneumothorax, it is common for intercostal tubes to be inserted by inexperienced junior doctors, fit poorly, leak, and become dislodged or infected; there is continuing confusion about suction, clamping, management of surgical emphysema, and when to seek specialist advice. Moreover, many patients find indwelling tubes uncomfortable and sometimes very painful.

Awareness has been increasing of the simplicity, effectiveness, and acceptability to patients of simple aspiration, ${ }^{12}$ and these advantages over tube drainage have been confirmed in a recent multicentre study. ${ }^{3}$ It is recognised that many patients with pneumothorax require no intervention at all. ${ }^{4}$ National guidelines for management of spontaneous pneumothorax now seem appropriate. The following plan has been drawn up on behalf of the British Thoracic Society after consultation with over 150 British respiratory physicians and thoracic surgeons. 
Fig I - Initial management of pneumothorax

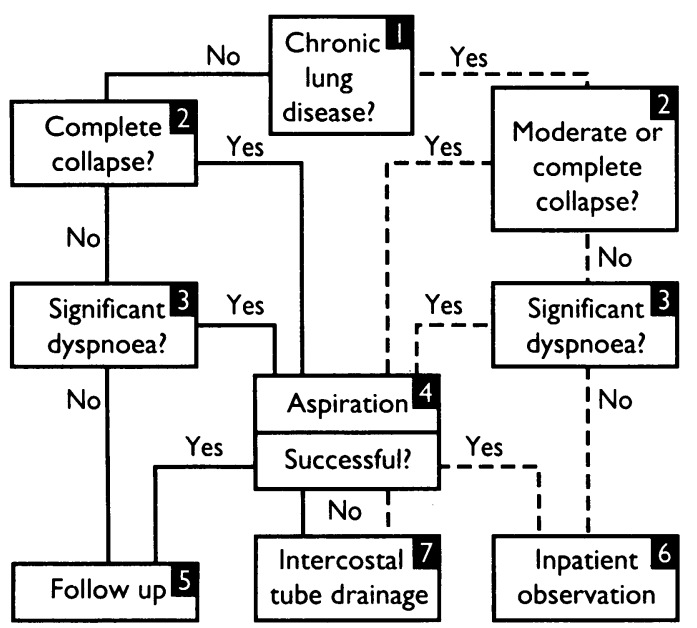

\section{Explanatory notes}

\section{Chronic lung disease}

In chronic lung disease-cystic, fibrotic, bullous, or emphysematous lung disease-respiratory compromise is commoner than with "simple" pneumothorax; drainage procedures are less successful, and referral to a respiratory specialist is more likely. Such patients must be observed overnight, whether or not they have had aspiration.

\section{Degree of collapse}

Small $=$ small rim of air around lung; Moderate $=$ lung collapsed halfway towards heart border; Complete =airless lung, separate from diaphragm -aspiration is necessary.

Tension =any pneumothorax with cardiorespiratory collapse. It requires immediate cannulation. It is rare.

\section{Significant dyspnoea}

This means an obvious deterioration in usual exercise tolerance. Aspiration is necessary, whatever the size of pneumothorax.

\section{Simple aspiration}

Infiltrate local anaesthetic down to the pleura, in the second intercostal space in the mid-clavicular line (the axillary aproach is an alternative). Using a cannula (French gauge 16 or larger and at least $3 \mathrm{~cm}$ long), enter the pleural cavity and withdraw the needle. Connect both the cannula and a $50 \mathrm{ml}$ syringe (Luer lock) to a three way tap, so that aspirated air can be voided.

Aspiration should be discontinued if resistance is felt or the patient coughs excessively, or more than 2.5 litres (that is, $50 \mathrm{ml}$ removed 50 times) is aspirated.

Repeat chest radiography in inspiration (an expiration film is unnecessary) in the $x$ ray department. If pneumothorax is now only small, or resolved, the procedure has been successful.

Note that failure to aspirate further may be due to the cannula being inadvertently withdrawn from the pleural cavity, or becoming kinked. If this is suspected, another attempt at aspiration should be considered.

\section{Follow up}

Arrange for a chest clinic appointment in 7-10 days. The patient must be given a discharge letter and told to attend again immediately in the event of noticeable deterioration. Air travel should be avoided until changes seen on radiographs have resolved.

\section{Inpatient observation}

Observe overnight. If patient is stable both clinically and radiologically, discharge with chest clinic appointment (see note 5).

\section{Intercostal tube drainage}

Throughout the procedure explain what is happening and reassure the patient. It may be advisable to premedicate with atropine to prevent a vasovagal reaction. If the patient is very anxious give a small dose of intravenous midazolam.

Always double check the correct side for insertion from the chest radiograph just before intubation. The usual site is the 4 th, 5 th, or 6 th intercostal space in the mid-axillary line. Position the patient supine with the head of the bed elevated $30^{\circ}$ and the patient's arm behind the head, resting away from the chest wall. Mark the site of insertion with a ballpoint or similar pen.

Since the drain may need to be in place for several days, sterile skin preparation and use of gloves are essential. Select a 20-24 French gauge (adult) drain and double check by dismantling and reassembling it. Ensure that all the connections fit tightly and that the underwater bottle containing sterile water is ready.

After palpating the intercostal space, raise an intradermal bleb of local anaesthetic, and then infiltrate the deeper tissues down to the parietal pleura, particularly around the periosteum on the upper surface of the lower rib (remember that the neurovascular bundle is on the lower surface). At first use a blue, and then green, needle with at least $10-20 \mathrm{ml}$ of either $0.5 \%$ bupivacaine plus adrenaline or $1 \%$ lignocaine. Aspirate intermittently, looking for air in the syringe to confirm that the pleural space has been entered.

When the local anaesthetic has worked, make an incision in the skin and subcutaneous fat; this should be less than $2 \mathrm{~cm}$, to ensure a tight fit of the drain. Then insert two horizontal sutures across the incision, leaving them loose for subsequent sealing of the wound on drain removal (complicated circular stitches are unnecessary). Using blunt dissection with forceps or a scalpel, make a wide tract through the intercostal muscles down to and through the parietal pleura.

Remember that the sharp metal point of a trocar does not cut or separate tissue and can be lethal if inserted forcibly. The trocar-drain assembly should slide in easily.

Once the tube is in the pleural space, withdraw the metal trocar $5 \mathrm{~cm}$ and advance the tube in an apical direction. Remove the trocar and connect to the underwater seal, securing it firmly with a suture (one loop through the skin and multiple ties in at least four places on the tube itself). Loop the tube and secure it again with plaster so that it cannot fall out. Ensure that the tube will not kink.

Prescribe adequate oral and intramuscular analgesia. 


\section{Fig 2 - Management of intercostal drains}

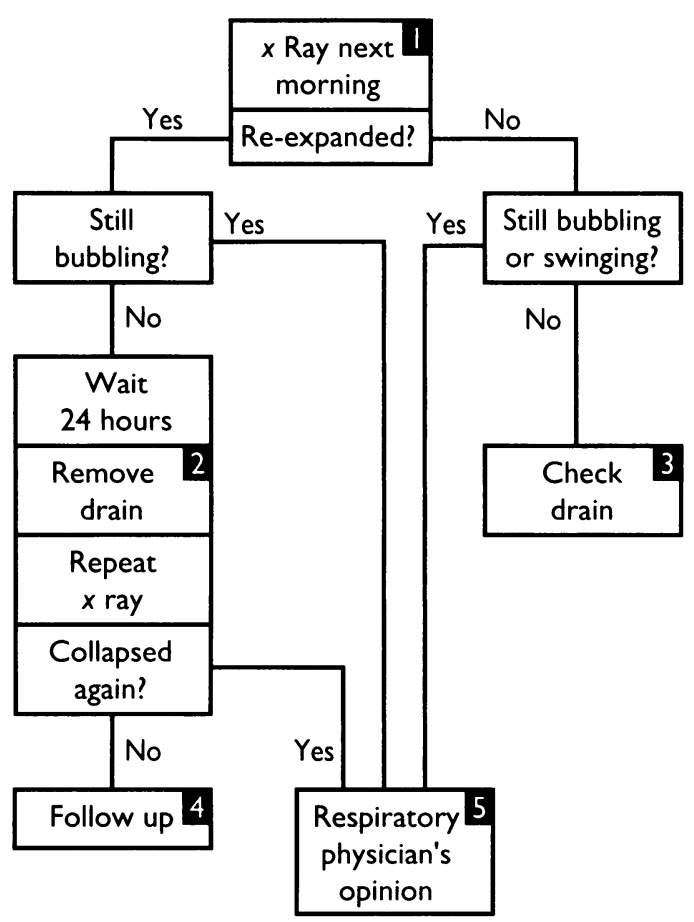

\section{Explanatory notes}

\section{Chest $x$ ray}

If the underwater seal is always kept below the level of the chest, clamping is unnecessary and potentially dangerous. As far as possible, an $x$ ray film should be taken in the department, rather than on the ward with a portable machine; an expiration film is unnecessary.

\section{Removal of chest drain}

Bubbling should have stopped for at least 24 hours.

Since some patients find tube removal unpleasant, consider premedication as above. After removing the suture that holds the drain in place, withdraw the tube while the patient holds his or her breath in full inspiration. Use the two remaining sutures to seal the wound.

\section{Check chest drain}

If the lung has not reinflated but there is no bubbling in the underwater bottle, then the tube is blocked or kinked - this can be corrected; or else the tube has become displaced-a replacement must be inserted through a clean incision.

\section{Follow up}

Arrange for a chest clinic appointment in 7-10 days. The patient must be given a discharge letter and told to attend again immediately in the event of noticeable deterioration. Air travel should be avoided until changes seen on radiographs have resolved.

\section{Respiratory physician's opinion}

Should advice from a specialist be required, transfer of continuing care is advisable. Important considerations in management are:

- assessing why re-expansion has not been achieved (for example, air leaking around the drain site, tube displaced or blocked, large persistent leak);

- the use of suction to re-expand the lung (this can be lengthy, requires appropriate equipment and pressure settings, influences how and where confirmatory radiographs are taken, and involves care from experienced nursing staff);

- whether early thoracic surgery would be appropriate (for example, failure of conservative measures, need to prevent recurrence);

- consideration of chemical pleurodesis in certain cases;

- management of surgical emphysema.
The diagrams and explanatory notes are designed to be incorporated into handbooks for medical units, and can be abbreviated (fig 1 plus notes 1-5) for display in casualty departments.

\section{Comment}

Many junior doctors, both in the casualty department and on medical teams, are becoming familiar with the technique of simple aspiration, which is quick to learn and easy to perform. Simple aspiration rather than an intercostal tube as the initial drainage procedure is greatly preferred by patients. As simple aspiration becomes more widely used, fewer intercostal tube drains will be necessary; in patients who require them, rapid re-expansion will be the exception. Early specialist advice is therefore likely to be needed for most intubated patients.

1 Raja OG, Lalor AJ. Simple aspiration of spontaneous pneumothorax. $\mathrm{Br} \mathcal{f}$ Dis Chest 1981;75:207-8.

2 Archer GJ, Hamilton AAD, Upadhyay R, Finlay M, Grace PM. Results of simple aspiration of pneumothoraces. Br $\mathcal{Y}$ Dis Chest 1985;79:177-82.

3 Harvey JE. Comparison of simple aspiration with intercostal drainage in the management of spontaneous pneumothorax. Thorax 1993;48:430-1. 4 Stradling P, Poole G. Conservative management of spontaneous pneumothorax Thorax 1966;21:145-9.

\section{Corrections}

Why have child pedestrian death rates fallen?

We regret that in figure 1 of this article by Ian Roberts (26 June, pp 1737-9) the labelling of symbols for traffic volume and fatality rate were inadvertently transposed. In fact, the closed symbols represented fatality rate and the open symbols traffic volume. Also, in the last paragraph of the introduction on $\mathrm{p} 1737$ and in the first paragraph on $\mathrm{p} 1738$ "children aged 0.4 years" should read "children aged $0-4$ years."
How can we best prolong life? Benefits of coronary risk factor reduction in non-diabetic and diabetic subjects

A typesetting error occurred in the earlier correction (26 June, p 1739) to this paper by John S Yudkin; the error occurred at some stage after the pages had been passed for publication by the editorial staff. The correction should have read: A typesetting error occurred in table IV of this paper by John S Yudkin (15 May, pp 1313-8). In the bottom line of the table (lowering systolic blood pressure $\geqslant 142 \mathrm{~mm} \mathrm{Hg}$ ) the entry in the second column of figures is wrong: it should read $0.83(-0.07$ to 1.79$)$, not $9.83(-0.07$ to $1 \cdot 79)$ 